\title{
Advanced Phase Reconstruction Methods Enabled by Four-Dimensional Scanning Transmission Electron Microscopy
}

Colin Ophus ${ }^{1 *}$, Tyler R Harvey ${ }^{2}$, Fehmi S Yasin², Hamish G Brown ${ }^{1}$, Philipp M Pelz ${ }^{1}$, Benjamin H Savitzky $^{1}$, Jim Ciston ${ }^{1}$, and Benjamin J McMorran ${ }^{2}$

1. NCEM, Molecular Foundry, Lawrence Berkeley National Laboratory, Berkeley, USA.

2. Department of Physics, University of Oregon, Eugene, USA.

*cophus@gmail.com

By converging an electron probe to small dimensions and measuring the diffracted signal, we can measure atomic-scale information about a sample's structure, orientation, deformation, composition, and more. A measurement of full 2D diffraction images over a 2D grid of probe positions produces a 4D dataset, referred to as four-dimensional scanning transmission electron microscopy (4D-STEM). With modern electron detectors, we can now measure thousands of diffraction patterns, which enables many new imaging methods. A large class of useful 4D-STEM imaging modes produce phase contrast images of specimens, for example differential phase contrast (DPC) and ptychography [1]. By combining 4DSTEM with phase plates, additional phase contrast imaging methods become possible [2]. The first topic of this talk will be to describe a related phase plate method called STEM holography (STEM-H) [3, 4], and its extension using ptychographic reconstruction. STEM-H is performed by using a diffraction grating in the probe forming aperture to produce multiple STEM beams, and then using a pixelated detector to measure the holographic fringe patterns, shown in Figure 1a. The primary advantage of STEM-H is that it produces phase contrast images on an absolute scale, which for example can be used to measure weak, extended electrostatic or electromagnetic fields as in Figures $1 \mathrm{~b}$. There, we see that for a conductive lacey carbon sample both the HAADF intensity and STEM-H phase shift show a similar decay into the vacuum. By contrast, for a semiconducting nanoparticle sample, we measure a phase shift due to an electrostatic field extending from the vacuum edge beyond the sample, which does not generate contrast in the HAADF channel. We have also extended STEM-H imaging to atomic resolution, shown in Figure 1c. STEM-H can quantitatively image the absolute phase shift of a sample, with the simple addition of a diffraction grating and large area, high speed direct electron detector.

The second topic covered in this talk will be phase reconstruction of the scattering matrix (S-matrix) from multiple 4D-STEM measurements. This formalism has the advantage of fully describing experiments where multiple scattering of the electron beam has occurred, and has recently been demonstrated experimentally [5]. We will discuss various methods to reconstruct the scattering matrix, and computational techniques to speed up the inversion procedure. STEM-H in particular is promising for S-matrix reconstruction, since it can measure the phase of all beams in a single measurement. Once the S-matrix has been reconstructed, it can be used to solve the structure of thick specimens as in [5], or to perform optical sectioning in a manner similar to [1]. To demonstrate the usefulness of this approach, we have performed multislice simulations of the S-matrix for a 4D-STEM experiment of a 3D stacked nanotube structure shown in Figure $2 \mathrm{a}$ and $\mathrm{b}$. Phase contrast images of 1 or 2 beams are shown in Figure $2 \mathrm{c}$, showing the angular resolution given by multiple beams. Figure $2 \mathrm{~d}$ shows optically sectioned phase contrast images constructed from the S-matrix at different heights, where the red arrows show the portions of the sample that are in focus. These advanced phase contrast imaging methods are only possible because of the high speed and high dynamic range detectors developed for use in 4D-STEM.[6] 


\section{References}

[1] H Yang et al., Nature Communications 7 (2016) 12532.

[2] C Ophus et al, Nature Communications 7 (2016), 10719.

[3] TR Harvey et al., Physical Review Applied 10 (2018) 061001.

[4] FS Yasin et al., Nanoletters 18 (2018) p 7118.

[5] HG Brown et al., Physical Review Letters 121 (2018) 266102.

[6] Work at the Molecular Foundry was supported by the Office of Science, Office of Basic Energy Sciences, of the U.S. Department of Energy under Contract No. DE-AC02-05CH11231. C Ophus, J Ciston and HG Brown acknowledge support of the DoE Early Career Research Program.
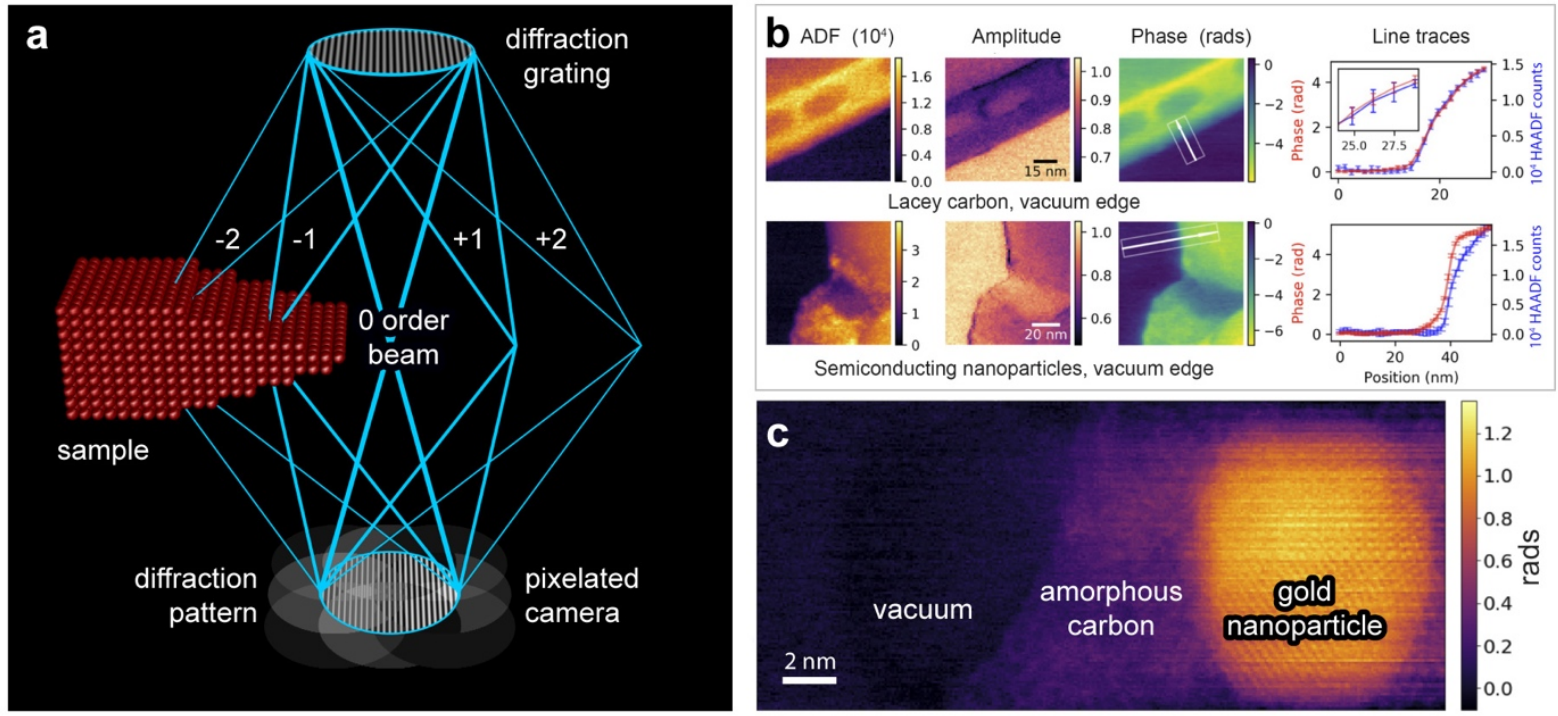

Figure 1. (a) STEM-H experimental geometry. (b) STEM-H phase measurements compared to HAADF for two samples. (c) STEM-H phase reconstruction at atomic resolution. Adapted from Refs [3] and [4].

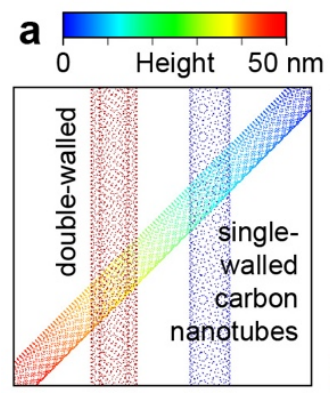

d

$\Delta f=3 \mathrm{~nm}$

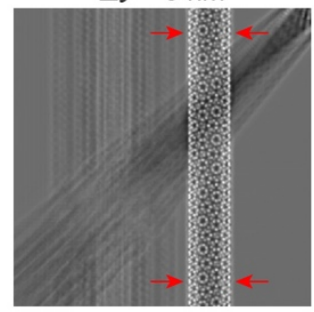

\section{b}

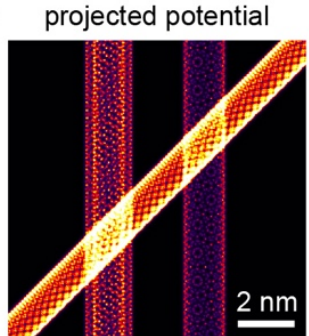

$\Delta f=15 \mathrm{~nm}$

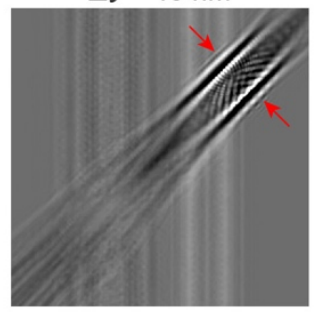

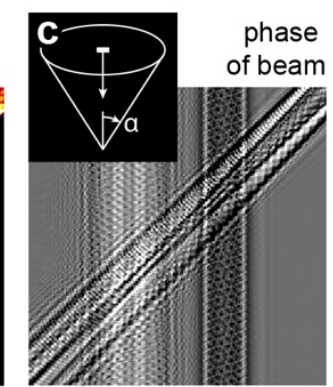

optical sectioning of S-matrix $\Delta f=26 \mathrm{~nm}$

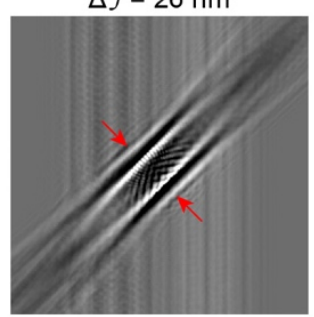

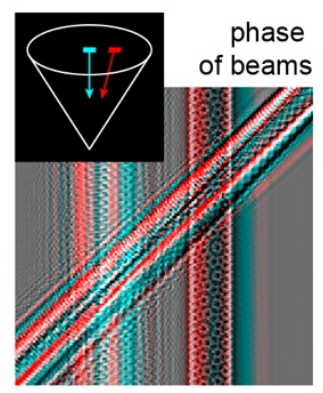

$\Delta f=37 \mathrm{~nm}$

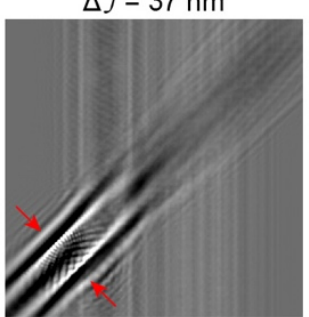

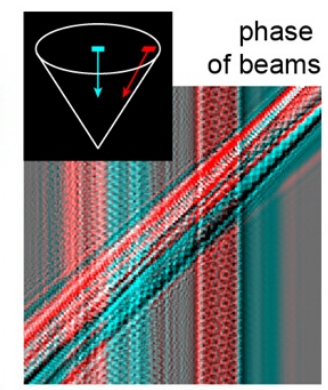

$\Delta f=49 \mathrm{~nm}$

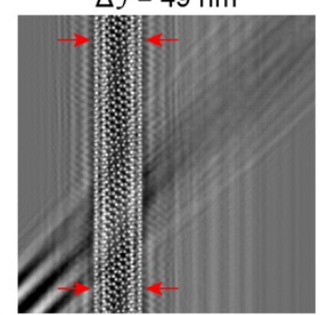

Figure 2. Multislice simulation of 4D-STEM S-matrix imaging of carbon nanotubes. (a) Atomic structure. (b) Projected potential. (c) Phase images of different beams taken from rows of the S-matrix. (d) Phase images computed by optical sectioning of the S-matrix. 